\title{
Pseudarthrosen nach Eingriffen am Fuß
}

Julia Evers, Sabine Ochman, Alexander Milstrey, Michael J. Raschke

\section{Einleitung}

Das Risiko, nach einer Fraktur eine Pseudarthrose zu entwickeln, wird laut amerikanischen Daten auf 5-10\% geschätzt [1,2]. Im klinischen Alltag wird, trotz unterschiedlicher Definitionen, bei ausbleibender Konsolidierung der Fraktur nach einer Zeitspanne zwischen 26 und 30 Wochen die Diagnose „Pseudarthrose“ gestellt [3]. Die Gründe einer ausbleibenden Frakturheilung sind oft multifaktoriell, eine Pseudarthrose kann auch trotz optimaler Osteosynthese, intaktem Weichteilmantel und Infektfreiheit entstehen.

In Deutschland werden pro Jahr bei steigender Inzidenz ca. 540000 Frakturen pro Jahr unter stationären Bedingungen operativ versorgt (ohne Berücksichtigung von Operationen an der Wirbelsäule) [4]. Analog zu der demografischen Entwicklung und der steigenden Lebenserwartung werden auch andere elektive orthopädische Operationen wie bspw. Arthrodesen immer häufiger durchgeführt [5].

Im Bereich des Fußes sind Rückfuß, Fußwurzel, Mittelund Vorfuß gleichermaßen von Pseudarthrosen betroffen [6]. Im Vordergrund der Behandlung im Bereich des Fußes stehen belastungsabhängige Beschwerden. Im Vergleich zu Pseudarthrosen langer Röhrenknochen wird durch Pseudarthrosen am Fuß die Stabilität zunächst nicht beeinträchtigt [6]. Die Belastbarkeit des Fußes, die Mobilität eines Menschen und die hiermit verbundene Lebensqualität hängen entscheidend vom Erfolg einer durchgeführten Frakturstabilisierung oder Arthrodese ab.

\section{Therapien}

Die Behandlung von Pseudarthrosen ist oft langwierig, kostenintensiv und unterliegt keinem generellen Standard. Es gibt verschiedene Therapiemöglichkeiten, Pseudarthrosen oder eine verzögerte Knochenheilung zu behandeln, um die Genesung eines Menschen zu beschleunigen und somit eine Wiederkehr ins Arbeits- und Sozialleben zu ermöglichen.

Die Kenntnis der Ursache der Pseudarthrose (biologisch mechanisch - infektiös) kann ein Schlüssel zur erfolgreichen Behandlung sein.
Die operative Revision einer Pseudarthrose bedeutet häufig ihre Resektion zusammen mit autologer Spongiosatransplantation und ist häufig langwierig, teuer und kann für den Patienten mit Schmerzen und schwerwiegenden Komplikationen assoziiert sein [7]. In den Fällen, bei denen eine große Instabilität oder Fehlstellung des betroffenen Knochens vorliegt oder Implantatversagen, ist jedoch die Revisionsoperation die einzige Möglichkeit.

Doch auch nach der Revision kann die Konsolidierung ausbleiben. Dies kann vielfache Gründe haben. Das Risiko für die Entwicklung einer Pseudarthrose wird mit gewissen Faktoren assoziiert, wie z. B. Stabilität des Konstrukts, Nikotinabusus, Diabetes, Osteoporose und Infektion [810]. Abhängig von der Lokalität der Pseudarthrose und dem Typ der Revision liegt die Wahrscheinlichkeit der Konsolidierung postoperativ bei 68-96\% [11].

Um einem Revisionseingriff mit entsprechender möglicher Komplikationsrate zu umgehen, können verschiedene Alternativen gewählt werden. Hier steht zum einen eine Gruppe aus verschiedenen Geräten zur Auswahl, die man unter der Überschrift „mechanische Knochenwachstumsstimulatoren“ zusammenfassen kann. Hierzu zählen bspw. der niedrig dosierte gepulste Ultraschall (LIPUS) und die fokussierte hochenergetische extrakorporale Stoßwelle (fhESWT).

LIPUS produziert Schallwellen, die mikromechanischen Stress auf die Fraktur oder Pseudarthrose ausüben. Dadurch entstehen zelluläre und molekulare Veränderungen, die die Knochenheilung vorantreiben können. LIPUS konnte bei verzögerten Frakturheilungen der langen Röhrenknochen und Pseudarthrosen gute Ergebnisse zeigen. Die Behandlung erfordert jedoch Disziplin vom Patienten, da sie durchschnittlich 20 min pro Tag für mehrere Monate durchgeführt werden muss [7].

Die extrakorporalen Stoßwellengeräte (fhESWT) produzieren hochenergetische Schallwellen, die durch elektrohydraulische, elektromagnetische oder piezoelektrische Mechanismen gepulste Stoßwellen generieren. In Tiermodellen konnten freie Radikale und Sauerstoffradikale nachgewiesen werden, die wiederum verschiedene Wachstumsfaktoren aktivierten [7]. Die fhESWT muss im Unterschied zur LIPUS unter ausreichender Analgesie ent- 

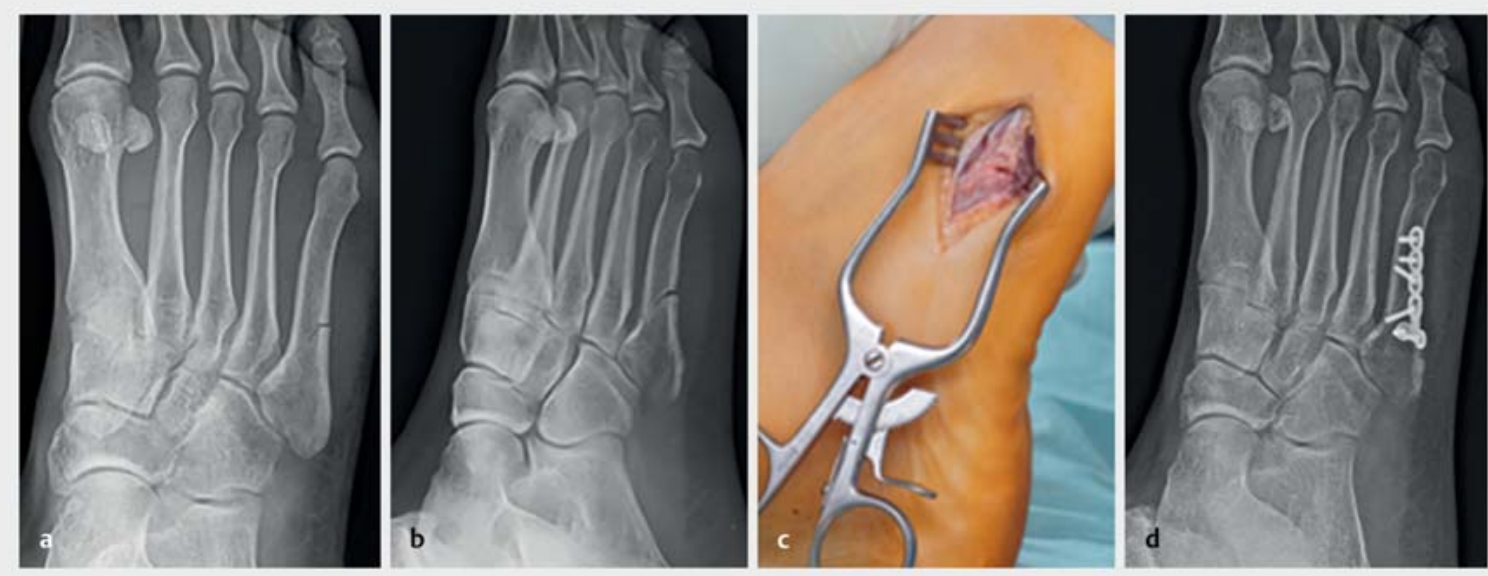

- Abb. 1 38-jährige Patientin, Umknicktrauma beim Tanzen. a Röntgen direkt nach Trauma. b Verlaufsröntgen 8 Wochen nach Trauma mit Nachweis einer verzögerten Frakturheilung. c Intraoperativer Befund. d Postoperatives Röntgen.

weder in Form eines Nervenblocks oder unter Vollnarkose erfolgen.

Um die Wirkung der fhESWT auf die Pseudarthrose möglichst effektiv zu gestalten, sollten verschiedene Kontraindikationen vermieden werden, die auf der Internetseite der Deutschsprachigen Internationalen Gesellschaft für Extrakorporale Stoßwellentherapie (DIGEST) nachzulesen sind [12].

Neben den mechanischen gibt es auch die biologischen Knochenwachstumsstimulatoren, die eine osteoinduktive Wirkung auf die Knochenheilung haben. Aktuell stehen die Wachstumsfaktoren BMP-2 und BMP-7 zur Verfügung, wobei eine Zulassung nur für BMP-7 für Tibiaschaftpseudarthrosen vorliegt. Die Anwendung von BMPs erfolgt jedoch zunehmend auch an anderen Lokalisationen und erzielt hier Durchbauungsraten von 85$90 \%$ [13]. Jedoch erfolgte die Anwendung aufgrund des Auftretens von Komplikationen mit größerer Vorsicht [14]. Weitere osteoinduktive Substanzen sind demineralisierte Knochenmatrix wie auch Thrombozytenkonzentrate (Platelet enriched Plasma, PRP). Ihre osteoinduktive Potenz ist jedoch im Vergleich mit BMP geringer. Über die Anwendung von BMP in der Fußchirurgie finden sich nur wenige Studien in der Literatur, jedoch mit guten Ergebnissen für Konsolidierung und Komplikationsraten [15].

\section{Pseudarthrosen bei Frakturen des Fußes}

Im Bereich des Fußes sind vor allem die Metatarsalia von dem Risiko der Entwicklung einer Pseudarthrose betroffen und hier vor allem das Os metatarsale $\mathrm{V}$ im proximalen Bereich.
Dies beruht sicherlich auch auf der Tatsache, dass die Therapie der Basisfrakturen am Mittelfußknochen V (MFK V) in der Literatur nicht einheitlich beschrieben wird und es sowohl Empfehlungen zur konservativen Therapie als auch zur perkutanen Schraubenosteosynthese als auch zur Plattenosteosynthese gibt mit unterschiedlichem Outcome für die Patienten [16-18]. Chuckpaiwong et al. sahen bei Jones-Frakturen und Schaftfrakturen des Metatarsale V genauso viele Refrakturen und Pseudarthrosen in der operativen wie in der konservativen Gruppe [18]. Mologne et al. konnten in einem Kollektiv aus Marinesoldaten nach operativer Therapie mittels Schraubenosteosynthese eine nur 7\%ige Pseudarthrosenrate nachweisen im Vergleich zur konservativen Gruppe mit einer Pseudarthrosenrate von 28\%. Die operativ behandelten Patienten waren früher wieder belastbar und auch die Refrakturrate war geringer [19].

Als prognostisch ungünstige Faktoren für die Entwicklung von Pseudarthrose im Bereich der Metatarsalia werden ein plantarer Frakturspalt von $>1 \mathrm{~mm}$ ( $\bullet$ Abb. 1), ein erhöhter Winkel zwischen Os metatarsale IV und V in der dorsoplantaren Aufnahme, eine vermehrte Lateraldeviation des Os metatarsale $\mathrm{V}$ in der $30^{\circ}$-Schrägaufnahme und ein hoher BMI beschrieben [20].

Ursache von Pseudarthrosen der übrigen Metatarsalia sind häufig übersehene Frakturen bei polytraumatisierten Patienten, aber auch nach elektiven Vorfußeingriffen kann es zu Pseudarthrosen in diesem Bereich des Fußes kommen. Dies führt häufig zu einer begleitenden Fehlstellung und konsekutiver Überlastung der benachbarten Metatarsalia [6].

Hinsichtlich der Beschwerden der Patienten erweisen sich diese als umso geringer, je basisnäher die Pseudarthrose 


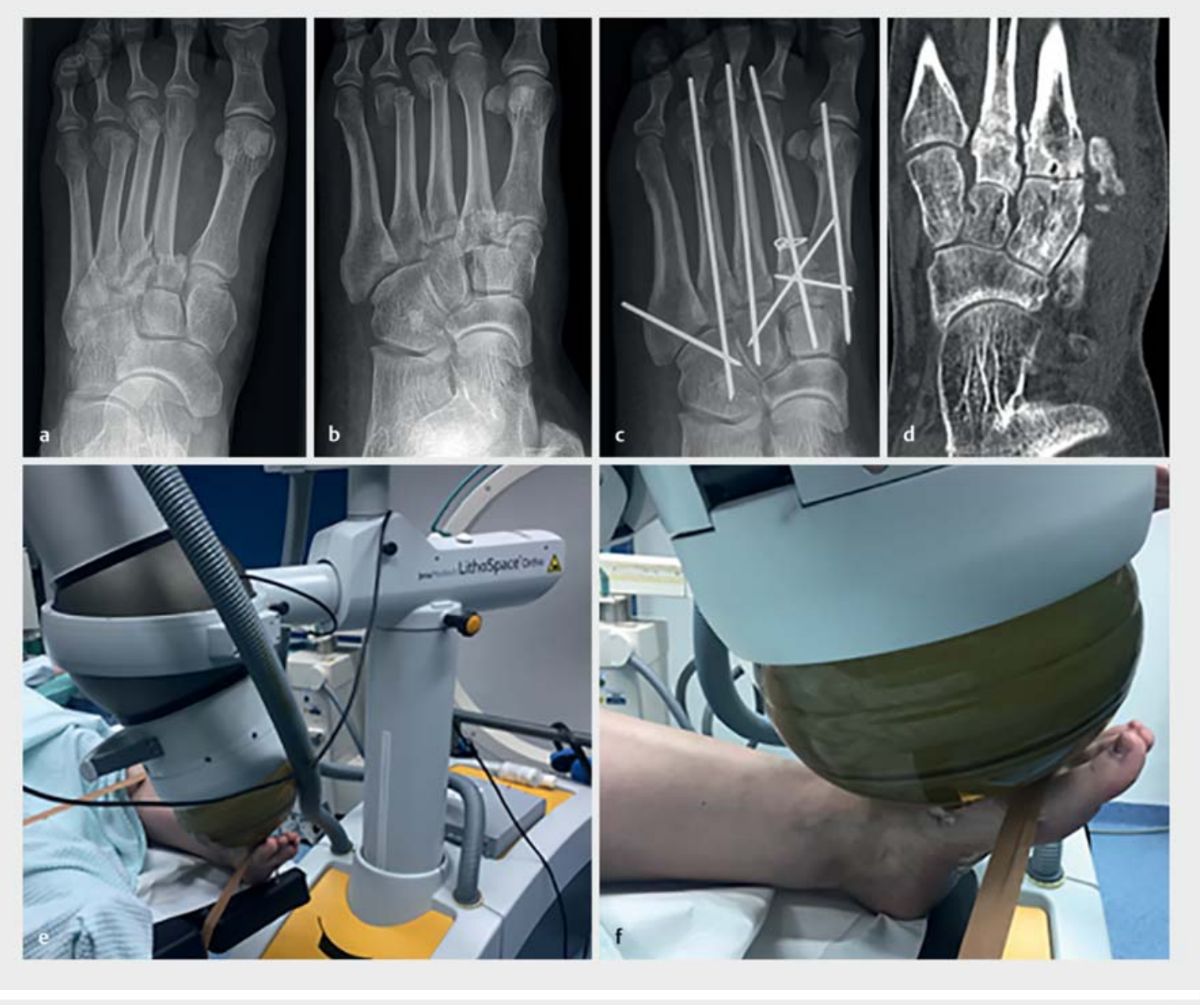

Abb. 2 44-jährige Patientin, Stolpersturz März 2018. a,b Röntgenbilder a.-p. und schräg mit Lisfranc-Luxationsfraktur und subkapitalen Frakturen MFK III und IV. c Röntgenkontrolle nach operativer Versorgung ex domo. d CT-Kontrolle 1 Jahr später mit Pseudarthrosen MFK-II- und -III-Basen. e,f Gezielte hochenergetische Stoßwellentherapie in Narkose. Quelle: Abb. a-d: Klinikum Rheine, Mathias-Spital, Rheine; Abb. e, f: Klinik und Poliklinik für Unfall-, Hand- und Wiederherstellungschirurgie, Universitätsklinikum Münster, Münster.

lokalisiert ist. Pseudarthrosen im distalen Metatarsalbereich sind meist mit einer erheblichen Beschwerdesymptomatik vergesellschaftet [6].

Bei isolierten Pseudarthrosen ohne größere Dislokation und einem Fraktur- oder Pseudarthrosenspalt $<5 \mathrm{~mm}$ ohne grobe Achs- oder Rotationsfehlstellung und Infektfreiheit kann die Stoßwellentherapie eine nicht invasive Alternative zur Revisionsoperation sein. In unserer Klinik wird die Stoßwelle mit der LitoSpaceOrtho ${ }^{\circledR}$ (JenaMedTech) appliziert. Die Behandlung erfolgt mit $3 \times 1000 \mathrm{Im}$ pulsen (Energieflussdichte $0,36 \mathrm{~m} / \mathrm{mm}^{2}$ ) bei $4 \mathrm{~Hz}$ und $23 \mathrm{kV}$, wobei die Applikation der Stoßwellen in einem $90^{\circ}$-Winkel zur Hautoberfläche und parallel zur Plattenausrichtung, sofern vorhanden, erfolgen soll. Als Ankopplungsmedium wird Ultraschallgel verwendet ( $\bullet$ Abb. 2 ).
Die Nachbehandlung erfolgt nach der Stoßwellenanwendung mit Abrollbelastung der behandelten Extremität für 6 Wochen in einer geeigneten Orthese.

Bei Vorliegen von schwerwiegenden Nebenerkrankungen kann es auch bei einer primär als „einfach“ zu wertenden Fraktur zu Komplikationen wie Pseudarthrosen mit Infekten kommen $[9,10]$, siehe $>$ Abb. 3 .

Auch im Bereich des Mittel- und Rückfußes können Pseudarthrosen entstehen, sie sind jedoch, wie die Frakturen selbst, relativ selten. Bei der Talushalsfraktur sind Pseudarthroseraten von bis zu $12 \%$ beschrieben, davon sind insbesondere die Hawkins-III-Frakturen betroffen [21] (॰ Abb. 4). 

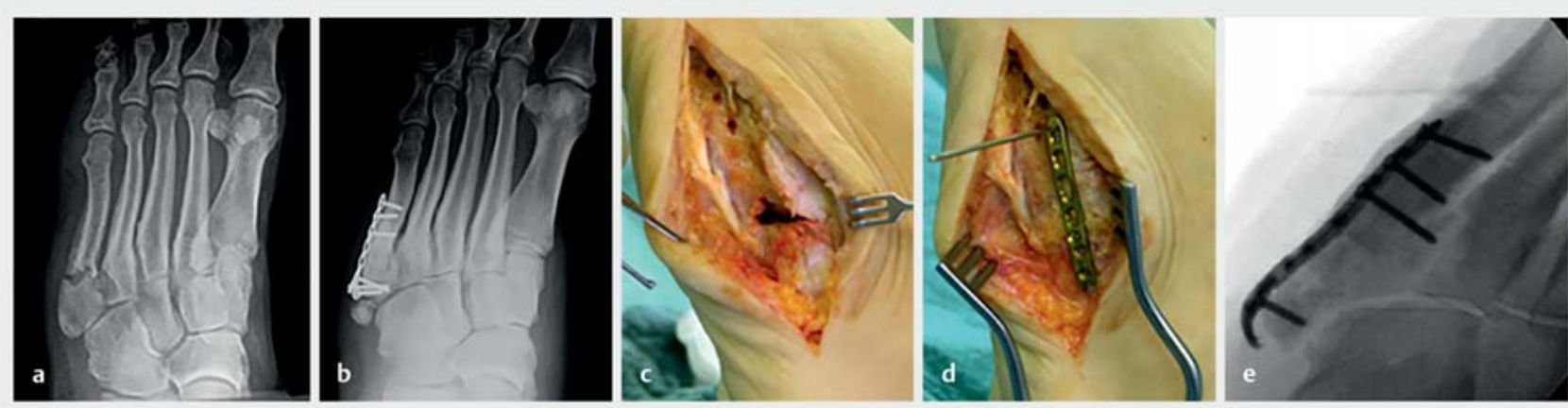

- Abb. 3 61-jähriger Patient, Typ-2-Diabetiker mit Polyneuropathie, erlitt im Dezember 2014 ein Supinationstrauma. Im Primärkrankenhaus erfolgte zunächst der Ausschluss einer Fraktur. Nach erneutem Trauma 6 Monate später mit Tritt in einen Nagel dann Röntgenkontrolle und Feststellen einer alten Jones-Fraktur. a Dann Plattenosteosynthese ex domo. Im Verlauf bei anhaltenden Beschwerden Vorstellung in unserer Klinik, hier erfolgte bei Diagnose einer periimplantären MFK-V-Basisfraktur (b) die Revision mit Plattenentfernung, Débridement, mikrobiologischen Proben (ohne Befund), Spongiosaanlagerung und Plattenosteosynthese (c-e). Drei Monate später stellte sich der Patient erneut vor wegen eines Infekts. Es erfolgte die Entfernung der Platte mit Débridement und Spülung. In den intraoperativ entnommenen Proben wurden Staphylococcus aureus und S. agalactiae nachgewiesen und resistogrammgerecht therapiert. Die Pseudarthrose war stabil und die Platte konnte ersatzlos entfernt werden.
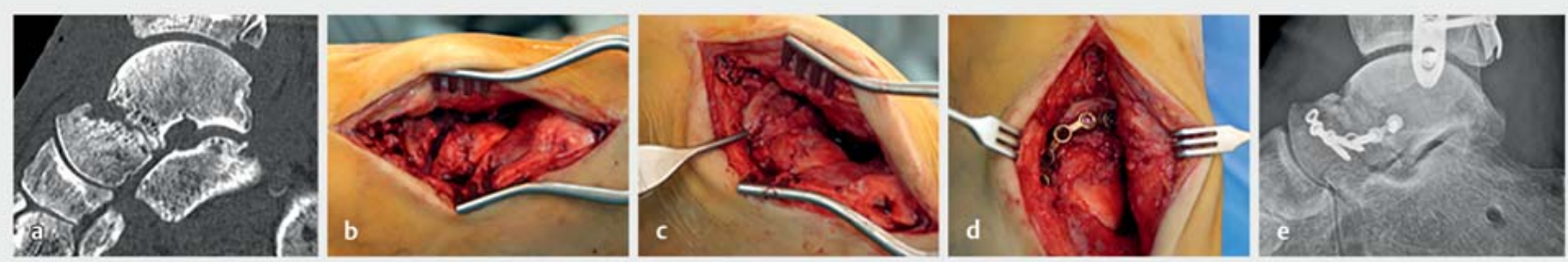

- Abb. 4 55-jähriger Patient mit u. a. Talushalsfraktur, Typ Hawkins III, die ex domo mittels 2-facher Schraubenosteosynthese behandelt wurde. Nach Nachweis einer Pseudarthrose (a), dann Reosteosynthese mit Débridement (b), Auffüllung des knöchernen Defektes mittels autogener Spongiosa (c) und Plattenosteosynthese (d, e).

Bei Frakturen im Bereich der Chopart-Linie sollte insbesondere auf die Längenverhältnisse der Fußsäulen und somit die Achs- und Längenverhältnisse der Knochen geachtet werden, was ebenso für Pseudarthrosen gilt. Sekundäre Vorfußabduktions- oder -adduktionsfehlstellungen können sich somit ergeben [22]. Pseudarthrosen der Fußwurzelknochen werden nur in Einzelfällen beschrieben und können eher aus übersehenen Frakturen entstehen ( $\triangleright$ Abb. 5).

\section{Pseudarthrosen bei Arthrodesen im Bereich des Fußes}

Auch bei Arthrodesen im Bereich des Fußes kann die Pseudarthrose eine schwerwiegende Komplikation darstellen, wenn sie Schmerzen und damit Mobilitätsein- schränkungen hervorruft. In diesen Fällen kann eine Revision unumgänglich sein.

Pseudarthrosenraten für Großzehengrundgelenksarthrodesen werden gemeinhin mit ca. 5\% angegeben [23], siehe Beispiel > Abb. 6.

Für Lisfranc-Arthrodesen liegen die Raten noch ein wenig höher, je nach Patientenkollektiv, zwischen 7 und 11\% [24]. Buda et al. konnten in ihrem Kollektiv nachweisen, dass für die Lisfranc-Arthrodesen die Verwendung einer Kombination von Kompressionsschrauben und Platten sowie die Anlage von autologer Spongiosa erhöhte Fusionsraten mit sich brachte [24].

Arthrodesen der Fußwurzelgelenke, wie des Talonavikulargelenks oder des Navikulokuneiformgelenks, die im 


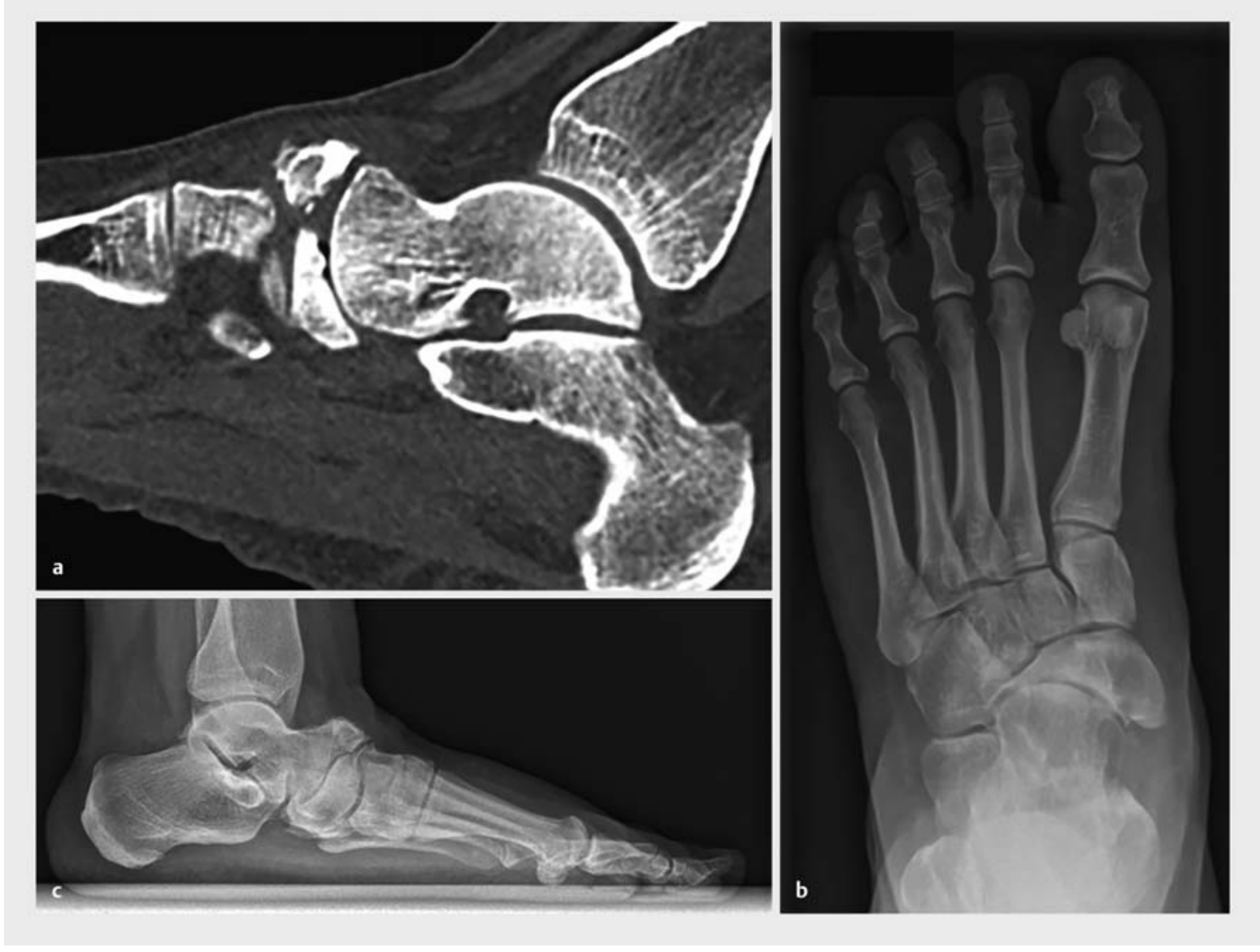

- Abb. 5 Eine 48-jährige Patientin stellte sich vor nach Sturz über einen Kübel 10 Jahre zuvor. Die Os-naviculare-Fraktur war primär übersehen und die Beschwerden der Patientin mit einer Pes-planovalgus-Deformität begründet worden (a). Durch die Fehlstellung des pseudarthrotisch ausgeheilten Os naviculare kommt es zu einer Protrusion des Talus, die für die Patientin in den Belastungsaufnahmen im Stehen weniger in einer Vorfußabduktionsfehlstellung (b) als in einer Pes-planus-Deformität (c) resultiert.

Zuge von Pes-planus- oder Pes-planovalgus-Deformitäten durchgeführt werden müssen, unterliegen ebenso der Gefahr der Entwicklung einer Pseudarthrose. Hier werden Raten zwischen 3 und $11 \%$ in der Literatur angegeben [25-27]. Ajis et al. wiesen darauf hin, dass in ihrem Kollektiv die Fusion der navikulokuneiformen Arthrodese durchschnittlich erst nach 21 Wochen eintrat [27]. Geduld kann sich in diesen Fällen also manchmal auszahlen, siehe Beispiel > Abb. 7.

\section{Fazit}

Pseudarthrosen können mit Schmerzen und Einschränkungen der Lebensqualität einhergehen. Das Risiko für die Entwicklung einer Pseudarthrose kann zum einen von patientenassoziierten Faktoren abhängig sein wie Diabetes, Durchblutungsstörungen, Vormedikation, Nikotin- und Alkoholabusus sowie fehlender Compliance. Aber auch operationstechnische Faktoren können Auslöser sein wie ein postoperativer Infekt, eine instabile Os- teosynthese, ein falsches Alignment oder eine unzureichende Vorbereitung der Knochenoberflächen bei der Arthrodese.

Bei Auftreten einer Pseudarthrose kann bei instabiler Osteosynthese, Infekten oder großen Knochendefekten die Revisionsoperation mit Spongiosatransplantation und erneuter Osteosynthese unumgänglich sein. Aber auch mit dieser Methode können Pseudarthrosen persistieren. Mechanische Knochenstimulatoren wie LIPUS oder fhESWT können eine Konsolidierung ohne Komplikationen, die mit einer Revisionsoperation vergesellschaftet sein können, herbeiführen. Biologische Knochenstimulatoren mit osteoinduktiver Wirkung können ebenfalls verwendet werden, sind aber Off-Label.

Im Bereich des Fußes geht eine Pseudarthrose im Gegensatz zu den langen Röhrenknochen nicht immer mit einem Verlust der Stabilität einher. Bei einer straffen Pseudarthrose, die keine oder wenig Beschwerden her- 

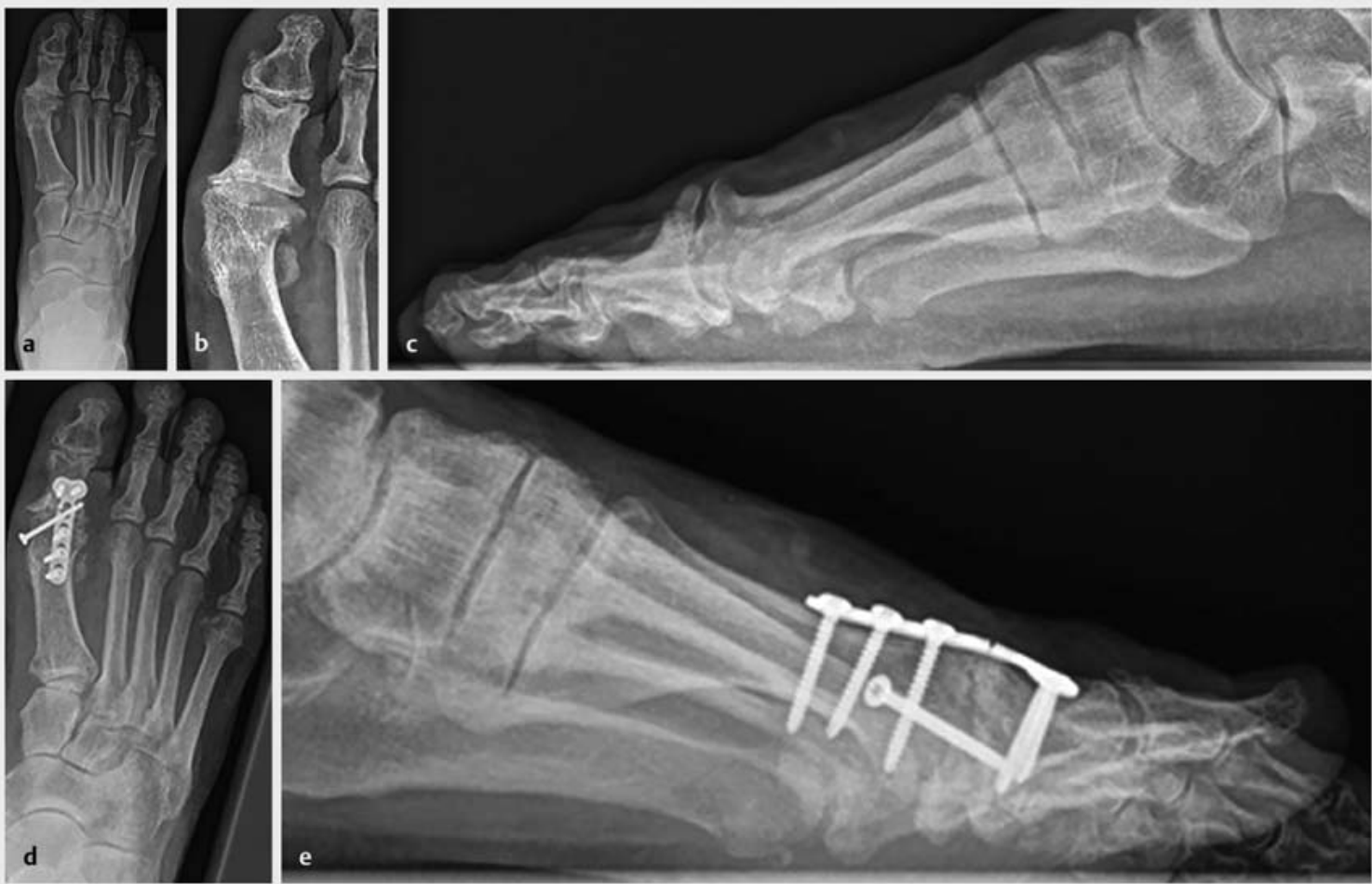

- Abb. 6 65-jährige Patientin mit Z. n. Hallux-valgus-Operation nach Keller/Brandes und nachfolgender Entwicklung einer sekundären Großzehengrundgelenksarthrose $(\mathbf{a}-\mathbf{c})$, die aufgrund des Alters der Patientin mittels Kompressionsschrauben- und Plattenosteosynthese behandelt wurde. Der weitere radiologische Verlauf ergab ein Implantatversagen (d,e) mit verzögerter Durchbauung. Da die Patientin keinerlei Beschwerden hatte unter Vollbelastung, ergab sich aus den Befunden keine Indikation zur Revision.
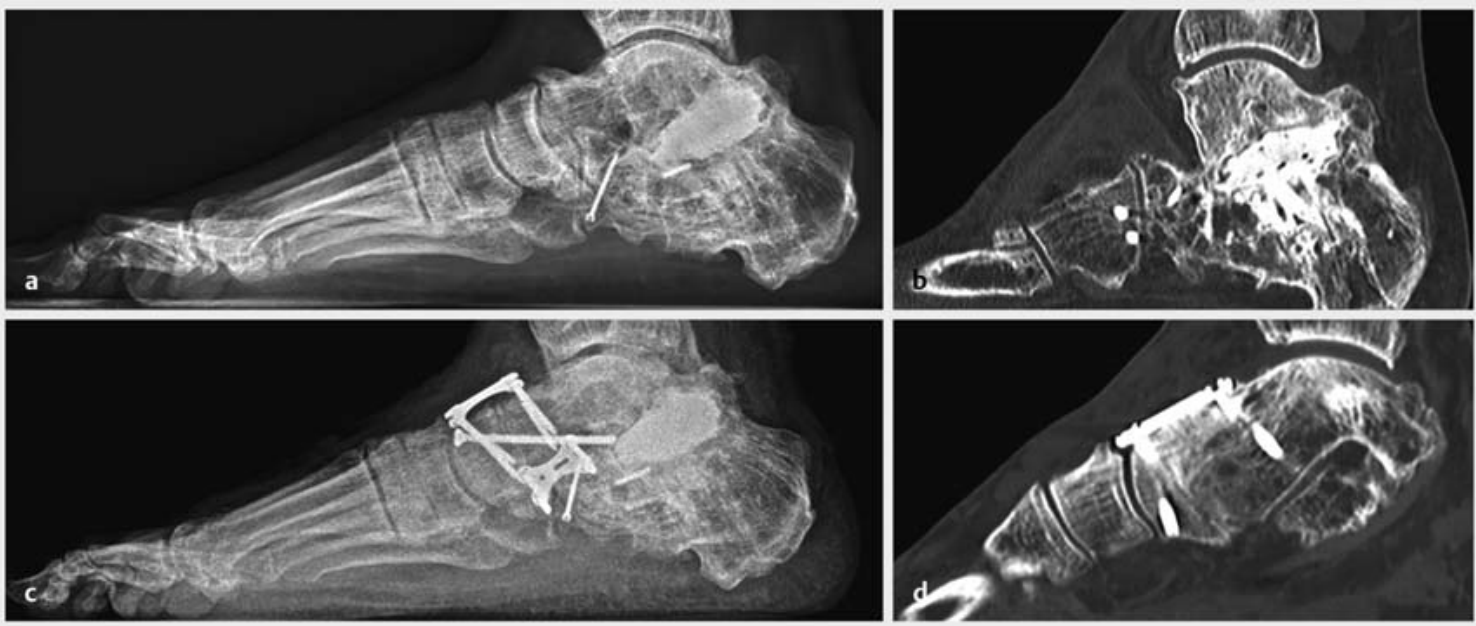

Abb. 7 37-jähriger Patient mit Typ-IV-Sanders-Fraktur des Kalkaneus und ex domo durchgeführter Arthrodese und ZementSpacer-Interposition zeigte Symptome von Anschlussarthrosen im Chopart-Gelenk. a, b Hier erfolgte eine Arthrodese mittels Spongiosaanlagerung und Plattenosteosynthese. Es entwickelte sich eine Pseudarthrose des Talonavikulargelenks (TN) und Kalkaneokuboidgelenks (CC) (c), die mittels Stoßwellentherapie und nachfolgender erneuter Revisionsarthrodese behandelt wurde. Im Verlauf zeigte das CC-Gelenk weiterhin eine ausbleibende Knochenheilung. $\mathbf{d}$ Mittels entsprechender Schuhversorgung waren jedoch die Beschwerden mild und das TN-Gelenk konsolidierte. 
vorruft, muss eine Revision nicht erfolgen oder kann mit entsprechenden orthopädieschuhtechnischen Vorrichtungen therapiert werden.

\section{Interessenkonflikt}

Die Autoren geben an, dass kein Interessenkonflikt besteht.

\section{Autorinnen/Autoren}
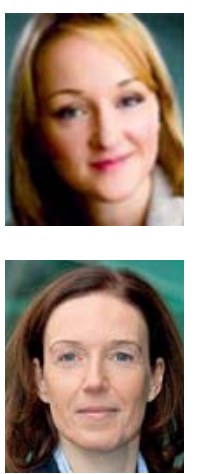

\section{Sabine Ochman}

Prof., geschäftsführende Oberärztin; Universitätsklinikum Münster, Klinik und Poliklinik für Unfall-, Hand- und Wiederherstellungschirurgie
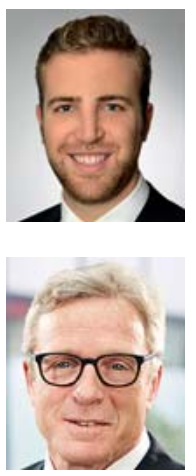

\section{Michael J Raschke}

Univ.-Prof., Direktor der Klinik, Universitätsklinikum Münster, Klinik und Poliklinik für Unfall-, Hand- und Wiederherstellungschirurgie

Korrespondenzadresse

Dr. Julia Evers

Klinik und Poliklinik für Unfall-, Hand- und Wiederherstellungschirurgie

Universitätsklinikum Münster

Albert-Schweitzer Campus 1, W1

48149 Münster

Tel.: 0251/56301

Fax: $0251 / 56318$

julia.evers@ukmuenster.de

\section{Literatur}

[1] Einhorn TA. Enhancement of fracture-healing. J Bone Joint Surg Am 1995; 77: 940-956

[2] Praemer A, Furner S, Rice DP. Musculoskeletal Conditions in the United States. Rosemont, IL: The American Academy of Orthopedic Surgeons; 1992: 85-124

[3] Bhandari M, Fong K, Sprague S et al. Variability in the definition and perceived causes of delayed unions and nonunions: a cross-sectional, multinational survey of orthopaedic surgeons. J Bone Joint Surg Am 2012; 94: e1091-e1096
[4] Garcia P, Domnick C, Lodde G et al. Operative Versorgung in Orthopädie und Unfallchirurgie. Unfallchirurg 2018; 121: 2029

[5] DESTATIS (Statistisches Bundesamt). Im Internet: www.destatis.de; https://www.destatis.de/DE/Home/_inhalt.html; Stand: 01.01.2019

[6] Wülker N. Operative und konservative Behandlungsmaßnahmen nach Fehlheilungen und Pseudarthrosen am Fuß. Trauma Berufskrankh 2003; 5 (Suppl. 1): S73-S76

[7] Buza JA III, Einhorn T. Bone healing in 2016. Clin Cases Miner Bone Metab 2016; 13: 101-105

[8] Wünschel M. Misserfolge nach Arthrodesen am Fuß. Orthopäde 2011; 40: 407-414

[9] Thevendran G, Wang C, Pinney SJ et al. Nonunion risk assessment in foot and ankle surgery: proposing a predictive risk assessment model. Foot Ankle Int 2015; 36: 901-907

[10] DiDomenico LA, Thomas ZM. Osteobiologics in foot and ankle surgery. Clin Podiatr Med Surg 2015; 32: 1-19

[11] Gebaur D, Mayr E, Orthner E et al. Low-intensity pulsed ultrasound: effects on nonunions. Ultrasound Med Biol 2005; 31: 1391-1402

[12] DIGEST (Deutschsprachige internationale Gesellschaft für Extrakorporale Stoßwellentherapie). Kontraindikationen. Im Internet: www.digest-ev.de; Stand: 23.07.2019

[13] Ronga M, Fagetti A, Canton G et al. Clinical applications of growth factors in bone injuries: experience with BMPs. Injury 2013; 44 (Suppl. 1): S34-S39

[14] Woo Ej. Adverse events after recombinant human BMP2 in nonspinal orthopaedic procedures. Clin Orthop Relat Res 2013; 471: 1707-1711

[15] Bibbo C, Nelson J, Ehrlich D et al. Bone morphogenetic proteins: indications and uses. Clin Podiatr Med Surg 2015; 32: 35-43

[16] Torg JS, Balduini FC, Zelko RR et al. Fractures of the base of the fifth metatarsal distal to the tuberosity. Classification and guidelines for non-surgical and surgical management. J Bone Joint Surg Am 1984; 66: 209-214

[17] Josefsson PO, Karlsson M, Redlund-Johnell I et al. Jones fracture. Surgical versus nonsurgical treatment. Clin Orthop 1994; 299: 252-255

[18] Chuckpaiwong B, Queen RM, Easley ME et al. Distinguishing Jones and proximal diaphyseal fractures of the fifth metatarsal. Clin Orthop Relat Res 2008; 466: 1966-1970

[19] Mologne TS, Lundeen JM, Clapper MF et al. Early screw fixation versus casting in the treatment of acute Jones fractures. Am J Sports Med 2005; 33: 970-975

[20] Lee KT, Park YU, Hegal $\mathrm{H}$ et al. Factors associated with recurrent fifth metatarsal stress fractures. Foot Ankle Int 2013; 34: 1645-1653

[21] Lindvall E, Haidukewych G, DiPasquale T et al. Open reduction and stable fixation of isolated, displaced talar neck and body fractures. J Bone Joint Surg Am 2004; 86: 2229-2234

[22] Ochman S, Evers ], Raschke MJ. Mittelfußverletzungen. Unfallchirurg 2011; 114: 883-892

[23] Roukis TS. Nonunion after arthrodesis of the first metatarsalphalangeal joint: a systematic review. J Foot Ankle Surg 2011; 50: 710-713

[24] Buda M, Hagemeijer NC, Kink S et al. Effect of fixation type and bone graft on tarsometatarsal fusion. Foot Ankle Int 2018; 39: 1394-1402

[25] Shenghui MA, Dan J. Isolated talonavicular arthrodesis. Foot Ankle Int 2016; 37: 905-908 
[26] Munoz MA, Augoyard R, Canovas F. Surgical treatment of hindfoot inflammatory diseases: 107 arthrodesis. Orthop Traumatol Surg Res 2012; 98 (Suppl.): S85-S90

[27] Ajis A, Geary N. Surgical technique, fusion rates and planovalgus foot deformity correction with naviculocuneiform fusion. Foot Ankle Int 2014; 35: 232-237
Bibliografie

DOI https://doi.org/10.1055/a-0944-8332

OP-JOURNAL 2019; 35: 339-346 @ Georg Thieme Verlag KG Stuttgart · New York ISSN 0178-1715 